\title{
Prevalencia de patología musculoesquelética asociada al síndrome de falla cardiaca
}

\author{
Prevalence of musculoskeletal pathology \\ associated with heart failure syndrome
}

\author{
Juan Manuel Sénior, Jesús Alberto Plata, Claudia María Navas \\ - Medellín (Colombia)
}

\section{Resumen}

Introducción: en pacientes con falla cardiaca se han descrito múltiples patologías asociadas, en especial las de origen musculoesquelético que pueden limitar la capacidad funcional, independiente de la función ventricular y deteriorar la calidad de vida de los pacientes, por lo que su detección permite realizar cambios en la prescripción del ejercicio y en el acondicionamiento aeróbico, aplicar medidas fisioterapéuticas y ergonómicas en los programas de rehabilitación cardiaca acorde con el diagnóstico específico.

Material y métodos: estudio descriptivo, de corte transversal y retrospectivo de los pacientes que ingresaron a la Clínica de Falla Cardiaca de la Universidad de Antioquia y el Hospital Universitario San Vicente de Paúl Fundación en un periodo de un año, con el objetivo de establecer la presencia de patología musculoesquelética.

Resultados: se ingresaron 151 pacientes durante el periodo de estudio. El 55.6\% fueron hombres y la mediana de edad fue de 68 años. La primera causa de falla cardiaca fue la enfermedad coronaria, seguida de la hipertensión arterial. El 33.1\% estuvieron en clase funcional de la Asociación de Corazón de Nueva York (NYHA) II y el 32.5\% en NYHA III. El 66.9\% de los pacientes que asisten a la Clínica de Falla Cardiaca no tenían actividad laboral.

Se demostró patología musculoesquelética en $31.8 \%$, siendo la más común la osteoartritis, bien sea de cadera o rodillas, con una frecuencia de $6.6 \%$, seguida de la tendinitis del manguito rotador $(4,6 \%)$. Otras alteraciones encontradas fueron dolor lumbar, dolor miofascial, neuropatía periférica, disfunción patelofemoral y síndrome de túnel carpiano. El 35.8\% de los pacientes se lograron vincular al programa de rehabilitación cardiaca y establecer un programa dirigido, de acuerdo con las comorbilidades encontradas.

Conclusiones: la patología osteomuscular en pacientes con falla cardiaca es frecuente, posiblemente por cambios metabólicos e hipoperfusión asociados a la falla cardiaca. El desacondicionamiento físico asociado aumenta el imbalance muscular y facilita síndromes por sobreuso como la tendinitis del manguito rotador. La vinculación a una Clínica de Falla Cardiaca con un programa de rehabilitación establecido, permitirá la detección temprana y la intervención adecuada durante el mismo de las patologías músculoesqueléticas asociadas haciendo que disminuya la discapacidad y mejore la calidad de vida de estos pacientes. (Acta Med Colomb 2014; 39: 233-237)

Palabras clave: ejercicio, comorbilidades no cardiacas, desórdenes musculoesqueléticos, rehabilitación cardiaca.

\footnotetext{
Abstract

Introduction: in patients with heart failure, multiple associated diseases have been described, especially those of musculoskeletal origin which may limit the functional capacity independent of ventricular function and deteriorate quality of life of patients; therefore, its detection allows changes in exercise prescription and aerobic conditioning, physiotherapy and to apply physiotherapeutic and ergonomic measures in cardiac rehabilitation programs according to the specific diagnosis.

Materials and methods: a descriptive, cross-sectional and retrospective cohort study of patients admitted to the Heart Failure Clinic of Antioquia University and the University Hospital San Vicente
}

Dr. Juan Manuel Senior Sánchez: FACP. Especialista en Medicina Interna, Cardiología y Cardiología Intervencionista. Coordinador Posgrado Cardiología Clínica e Intervencionista, Universidad de Antioquia, Hospital Universitario San Vicente de Paúl Fundación; Dr. Jesús Alberto Plata: MSc. Especialista en Medicina Física y Rehabilitación, Magister en Ciencias Clínicas. Coordinador Posgrado Medicina Física y Rehabilitación, Universidad de Antioquia; Dra. Claudia María Navas R.: Especialista en Medicina Física y Rehabilitación. Profesora Programa Posgrado Cardiología Clínica, Universidad de Antioquia. Medellín (Colombia).

Correspondencia. Dr. Juan Manuel Senior Sánchez. Medellín (Colombia).

E-mail: mmbt@une.net.co

Recibido: 28/VII/2013 Aceptado: 1/VIII/2014 
de Paul Foundation over a period of one year with the aim of establishing the presence of musculoskeletal pathology.

Results: 151 patients were admitted during the study period. $55.6 \%$ were men and the median age was 68 years. The leading cause of heart failure was coronary heart disease, followed by hypertension. $33.1 \%$ were in functional class II of the New York Heart Association (NYHA) and $32.5 \%$ in NYHA III. $66.9 \%$ of patients attending the heart failure clinic did not have work activity.

Musculoskeletal pathology was demonstrated in $31.8 \%$, being osteoarthritis the most common, either of hip or knee, with a frequency of $6.6 \%$, followed by rotator cuff tendinitis (4.6\%). Other alterations found were low back pain, myofascial pain, peripheral neuropathy, patellofemoral dysfunction and carpal tunnel syndrome. $35.8 \%$ of patients were able to link the cardiac rehabilitation program and establish a program targeted according co-morbidities found.

Conclusions: musculoskeletal pathology in patients with heart failure is common, possibly due to metabolic changes and hypoperfusion associated with heart failure. Physical deconditioning associated increases muscle imbalance and facilitates overuse syndromes such as rotator cuff tendinitis. Linking of a heart failure clinic with an established rehabilitation program will allow early detection and appropriate intervention during it of the musculoskeletal pathologies associated, reducing in this way disability and improving the quality of life of these patients. (Acta Med Colomb 2014; 39: 233-237)

Keywords: exercise, noncardiac comorbidities, musculoskeletal disorders, cardiac rehabilitation.

\section{Introducción}

La falla cardiaca es una enfermedad crónica, progresiva e incapacitante que se presenta como el estado final de diversas patologías cardiovasculares tales como la hipertensión arterial y la enfermedad coronaria. Tiene una alta prevalencia en la población general; entre los pacientes con hipertensión arterial, 76\% de los hombres y $79 \%$ de las mujeres terminan en falla cardiaca, luego de un seguimiento a 32 años (1). Aproximadamente 5 millones de personas en los Estados Unidos tienen falla cardiaca y cada año se presentan aproximadamente 670000 nuevos casos en personas mayores de 45 años y 274000 muertes están relacionadas con el síndrome, siendo la causa en más de 56000 casos (1). La edad avanzada es un factor de riesgo mayor para el desarrollo de falla cardiaca crónica. La incidencia es de $0.2 \%$ entre los 45 y 55 años, y se duplica con cada década de incremento (1).

La intolerancia al ejercicio de los pacientes con falla cardiaca crónica fue considerado hasta hace poco como un síntoma peligroso que precedía a su descompensación, lo cual produce inactividad, desacondicionamiento y genera la aparición de otras complicaciones. Los programas bien diseñados, basados en el ejercicio pueden mejorar la capacidad funcional y la calidad de vida en pacientes con falla cardiaca crónica (2), pero éste a su vez se puede ver entorpecido por patologías asociadas, dado que pueden interferir en el desarrollo adecuado de los diversos tipos de actividad física propuestos dentro de un programa de ejercicio monitorizado. Adicionalmente, las comorbilidades pueden comportarse como factores predictores, relacionadas con un incremento en la tasa de hospitalizaciones por descompensación y estancias hospitalarias prolongadas (3). Los beneficios del entrenamiento han sido atribuidos en particular a adaptaciones periféricas, incluyendo mejoría en la capacidad oxidativa del músculo esquelético $(4,5)$. El entrenamiento con ejercicio está asociado con reducción en la resistencia periférica y en menor proporción, con reducción de los volúmenes ventriculares, de la fracción de eyección y de la cardiomegalia (5).

En pacientes con falla cardiaca se han descrito múltiples patologías asociadas, en especial las de origen musculoesquelético como la osteoartritis, con una prevalencia de $16 \%$, compromiso de la columna vertebral como la espondilólisis y el dolor lumbar en $7 \%$, la osteoporosis en $5 \%$ y otras complicaciones de origen no cardiaco como las patologías tiroideas, la depresión, los trastornos de ansiedad y de personalidad, la enfermedad pulmonar obstructiva crónica y diferentes patologías oculares (3). Un estudio de Shen y colaboradores (6), realizado en 250 pacientes con cardiopatía, con o sin falla cardiaca, quienes ingresaron a un programa de rehabilitación, demostró que $62 \%$ de ellos tenían problemas musculoesqueléticos, siendo más frecuentes los que afectaban la columna lumbar (47\%), seguidos de la rodilla (32\%) y hombros (14\%), y $29 \%$ de los pacientes tenían dolor importante con la actividad física, lo que en conjunto limitaba su capacidad funcional.

El conocimiento de la presencia de patología musculoesquelética en los pacientes con falla cardiaca es importante en la aplicación de los programas de rehabilitación cardiaca dado que permite tener un diagnóstico integral de los factores que inciden en el estado funcional y la calidad de vida del paciente con falla cardiaca, realizar cambios en la prescripción del ejercicio y en el acondicionamiento aeróbico de acuerdo con la patología musculoesquelética encontrada y aplicar medidas fisioterapéuticas y ergonómicas que mejoren el desempeño funcional del paciente con falla cardiaca dependiendo del diagnóstico musculoesquelético (7). 


\section{Material y métodos}

Se realizó un estudio descriptivo, de corte transversal y retrospectivo de los pacientes que ingresaron a la Clínica de Falla Cardiaca de la Universidad de Antioquia y el Hospital Universitario San Vicente de Paúl Fundación en un periodo de un año. A la consulta de falla cardiaca asiste un cardiólogo, la enfermera del programa y un fisiatra con entrenamiento en rehabilitación en falla cardiaca, en la cual se realiza una evaluación integral, se define su opción terapéutica y la posibilidad de ingresar al programa, el cual incluye rehabilitación cardiaca. Durante esta evaluación el fisiatra es quien evalúa y registra la presencia de patologías musculoesqueléticas asociadas. Se tuvieron en cuenta la edad, el sexo, la clase funcional según la clasificación de la New York Heart Association (NYHA) (8), la etiología de la falla cardiaca y su ocupación en ese momento; además la presencia de patología musculoesquelética y si se benefició o no de ingresar al programa de rehabilitación cardiaca.

\section{Análisis estadístico}

Las variables cuantitativas se muestran con promedios y desviaciones estándar, o medianas y el rango intercuartil. Las variables cualitativas por medio de frecuencias. Se hizo un análisis explorador de la asociación entre clase funcional y patología musculoesquelética, calculando los OR y sus intervalos de confianza por medio de tablas dos por dos, con el software EpiDat 3.5.

\section{Resultados}

Se revisaron 151 historias clínicas de pacientes que asistieron por primera vez a la consulta de Clínica de Falla Cardiaca. Las características de los pacientes se describen en la Tabla 1. La mediana de la edad fue de 68 años con un rango entre los 20 y 88 años, los hombres fueron el $55.6 \%$. De acuerdo con la etiología de la falla cardiaca, la primera causa fue la enfermedad coronaria, seguida de la hipertensión arterial, las valvulopatías, la idiopática y la mixta. La mayor parte de los pacientes estuvieron en clase funcional
Tabla 1. Características epidemiológicas y clínicas de un grupo de pacientes con Falla Cardiaca del Hospital Universitario San Vicente de Paúl Fundación.

\begin{tabular}{|c|c|c|}
\hline \multirow[t]{2}{*}{ Características } & \multicolumn{2}{|l|}{ Total } \\
\hline & $\mathbf{N}$ & $\%$ \\
\hline $\begin{array}{l}\text { Sexo } \\
\quad \begin{array}{l}\text { Masculino } \\
\text { Femenino }\end{array}\end{array}$ & $\begin{array}{l}84 \\
67\end{array}$ & $\begin{array}{l}55.6 \\
44.4\end{array}$ \\
\hline $\begin{array}{l}\text { Edad } \\
\quad \text { Media (rango) }\end{array}$ & $68(20-88)$ & \\
\hline $\begin{array}{l}\text { Etiología } \\
\text { Enfermedad coronaria } \\
\text { Valvulopatía } \\
\text { Hipertensiva } \\
\text { Fiebre reumática } \\
\text { Enfermedad de Chagas } \\
\text { Idiopática } \\
\text { Isquémica e hipertensiva }\end{array}$ & $\begin{array}{c}42 \\
28 \\
39 \\
3 \\
1 \\
21 \\
17\end{array}$ & $\begin{array}{c}27.8 \\
18.5 \\
25.8 \\
2 \\
0.7 \\
13.9 \\
11.3\end{array}$ \\
\hline $\begin{array}{l}\text { Clase funcional } \\
\text { I } \\
\text { II } \\
\text { III } \\
\text { IV }\end{array}$ & $\begin{array}{l}40 \\
50 \\
49 \\
12\end{array}$ & $\begin{array}{c}26.5 \\
33.1 \\
32.5 \\
7.9\end{array}$ \\
\hline $\begin{array}{l}\text { Actividad ocupacional } \\
\text { Sí } \\
\text { No }\end{array}$ & $\begin{array}{c}50 \\
101\end{array}$ & $\begin{array}{l}33.1 \\
66.9\end{array}$ \\
\hline $\begin{array}{l}\text { Programa de rehabilitación } \\
\text { Sí } \\
\text { No }\end{array}$ & $\begin{array}{l}54 \\
97\end{array}$ & $\begin{array}{l}35.8 \\
64.2\end{array}$ \\
\hline $\begin{array}{l}\text { Patología musculoesquelética } \\
\text { Sí } \\
\text { No }\end{array}$ & $\begin{array}{c}48 \\
103\end{array}$ & $\begin{array}{l}31.8 \\
68.2\end{array}$ \\
\hline
\end{tabular}

II y III de la NYHA. El 66.9\% de los pacientes que asisten a la Clínica de Falla Cardiaca no tienen actividad laboral.

Se demostró patología musculoesquelética en $31.8 \%$ de los pacientes al momento del ingreso a la Clínica de Falla Cardiaca. La patología musculoesquelética más común fue la osteoartritis, bien sea de cadera o rodillas, con una frecuencia de $6.6 \%$ (Tabla 2), seguida de la tendinitis del manguito rotador en siete casos con una frecuencia de $4,6 \%$. El dolor lumbar, el dolor miofascial, en la musculatura de la cintura escapular, del cuadrado lumbar y de los glúteos

Tabla 2. Distribución de la patología musculoesquelética y género de un grupo de pacientes con falla cardiaca del Hospital Universitario San Vicente de Paúl Fundación.

\begin{tabular}{|c|c|c|c|c|c|c|}
\hline Patología & Masculino & $\%$ & Femenino & $\%$ & Total & $\%$ \\
\hline Tendinitis del manguito rotador & 4 & 2.6 & 3 & 2 & 7 & 4.6 \\
\hline Osteoartritis & 8 & 5.3 & 2 & 1.3 & 10 & 6.6 \\
\hline Dolor lumbar & 3 & 2 & 2 & 1.3 & 5 & 3.3 \\
\hline Dolor cervical & 0 & 0 & 1 & 0.7 & 1 & 0.7 \\
\hline Dolor miofascial & 4 & 2.6 & 1 & 0.7 & 5 & 3.3 \\
\hline Disfunción patelofemoral & 0 & 0 & 2 & 1.3 & 2 & 1.3 \\
\hline Lesiones de nervio periférico & 3 & 2 & 2 & 1.3 & 5 & 3.3 \\
\hline Síndrome del túnel del carpo & 0 & 0 & 2 & 1.3 & 2 & 1.3 \\
\hline Otros & 6 & 4 & 5 & 3.3 & 11 & 7.3 \\
\hline Sanos & 56 & 37.1 & 47 & 31.1 & 103 & 68.2 \\
\hline Total & 84 & 55.6 & 67 & 44.3 & 151 & 100 \\
\hline
\end{tabular}


tanto mayor como medio, se encontró en $3.3 \%$ de los casos, cada uno. Las neuropatías periféricas, dentro de las cuales hay casos de polineuropatía diabética y lesiones del nervio fibular común y del ciático, se presentaron en $3.3 \%$ de los casos. En el último lugar están la disfunción patelofemoral y el síndrome del túnel del carpo con dos casos cada patología, que fueron descritos únicamente en el sexo femenino. El $35.8 \%$ de los pacientes se lograron vincular al programa de rehabilitación cardiaca y establecer un programa dirigido, de acuerdo con las comorbilidades encontradas.

De acuerdo con la clase funcional es más común encontrar patologías musculoesqueléticas en los pacientes que tienen mejor clase funcional, es decir en la clase funcional de la NYHA II y III con $39.6 \%$ y $29.2 \%$ respectivamente (Tabla 3). Al realizar la exploración de la asociación entre la clase funcional de los pacientes y la presencia o no de patología musculoesquelética no se encontraron relaciones significativas, incluyendo los valores de los intervalos de confianza (Tabla 4).

\section{Discusión}

Las patologías musculoesqueléticas asociadas al síndrome de falla cardiaca, son comorbilidades que se presentan con bastante frecuencia dado el grupo etareo afectado que ameritan una intervención adecuada y oportuna en un programa de rehabilitación cardiaca. Encontramos presencia de alteraciones musculoesquelética en $31.8 \%$ de los casos, lo que potencialmente se convierte en una limitante para el programa de rehabilitación, con obvias implicaciones en la capacidad funcional. En la literatura mundial son escasos

Tabla 3. Patología musculoesquelética y clase funcional de un grupo de pacientes con falla cardiaca del Hospital Universitario San Vicente de Paúl Fundación.

\begin{tabular}{|c|cc|cc|c|}
\hline \multicolumn{7}{|c|}{ Patología musculoesquelética } \\
\hline Clase funcional & Ausente & $\mathbf{\%}$ & Presente & $\%$ & Total \\
\hline I & 29 & 28.1 & 11 & 22.9 & 40 \\
II & 31 & 30.1 & 19 & 39.6 & 50 \\
III & 35 & 34 & 14 & 29.2 & 49 \\
IV & 8 & 7.8 & 4 & 8.3 & 12 \\
\hline Total & $\mathbf{1 0 3}$ & $\mathbf{1 0 0}$ & $\mathbf{4 8}$ & $\mathbf{1 0 0}$ & $\mathbf{1 5 1}$ \\
\hline
\end{tabular}

Tabla 4. Asociación entre la clase funcional y la patología musculoesquelética de un grupo de pacientes con falla cardiaca del Hospital Universitario San Vicente de Paúl Fundación.

\begin{tabular}{|c|c|c|}
\hline Clase funcional & OR & IC 95\% \\
\hline I & Referente & \\
II & 1.61 & $0.65-3.9$ \\
III & 1.05 & $0.42-2.67$ \\
IV & 1.31 & $0.33-5.3$ \\
\hline
\end{tabular}

los reportes de las comorbilidades no cardiacas de la falla cardiaca crónica. Braunstein y colaboradores (3) reportan una prevalencia de osteoartritis de $16 \%$, de problemas lumbares de $7 \%$ y de osteoporosis de $5 \%$, en una población de 122630 personas mayores de 65 años con falla cardiaca crónica. En nuestro estudio la osteoartritis también fue la patología más común, aunque con una frecuencia de $6.6 \%$, que es mucho menor a la descrita anteriormente, lo mismo sucede con el dolor lumbar, ya que en este estudio su prevalencia fue de $3.3 \%$ únicamente.

En la población general, la osteoartritis tiene una prevalencia de $12.5 \%$ (9), la tendinitis del manguito rotador entre 5 y $25 \%$ (10) y en el caso dolor miofascial es de un $14.4 \%$ (11), el dolor lumbar tiene una prevalencia de 20-30\% (12). Por lo tanto, la asociación no expresa una relación de causa-efecto, sino simplemente la presentación de patologías con alta tasa de prevalencia en la población de edad avanzada, con una mediana de edad de 68 años en nuestro reporte. Adicionalmente, en esta investigación sólo se estudió un grupo de pacientes que asisten a una Clínica de Falla Cardiaca y por lo tanto no son comparables a los resultados en estudios poblacionales.

Las causas etiológicas del dolor de origen musculoesquelético en el síndrome de falla cardiaca crónico, se propone que los cambios pueden darse en el metabolismo oxidativo de la fibra muscular, debido a: función alterada en las mitocondrias, alteraciones en la miofibrilla que hace que el acople de las estructuras sea menos sensible al ingreso de calcio a la célula; esto explicaría la alteración en la economía muscular, y tiene como consecuencia la intolerancia progresiva al ejercicio y una discapacidad física mayor que presentan los pacientes con falla cardiaca (12-14). Por otro lado se debe tener en cuenta también las consecuencias del síndrome de desacondicionamiento físico que va de la mano con el reposo secundario a la intolerancia de la actividad física, dando como resultado diferentes grados de imbalance muscular en sistemas agonista y antagonista que facilitan los síndromes por sobreuso como la tendinitis del manguito rotador y esto se puede ver claramente en la mayor presentación de casos de patologías musculoesqueléticas en las clases funcionales que toleran mejor la actividad física y que por lo tanto pueden desarrollar más actividades que los pacientes en clase funcional IV. Por último no se deben olvidar las posibilidades que tiene un paciente de ser evaluado por un grupo multidisciplinario, en este caso por cardiología y rehabilitación, donde es posible detectar no sólo este tipo de comorbilidades, sino también ofrecer el acceso a un programa protocolizado de rehabilitación cardiaca.

Los programas de rehabilitación cardiaca deben tener muy en cuenta el compromiso musculoesquelético para diseñar protocolos que no sólo realicen programas de reacondicionamiento general sino que se traten a su vez problemas específicos que permitan mayor adherencia y por ende mejores resultados. 


\section{Conclusión}

La patología osteomuscular en pacientes con falla cardiaca es frecuente; posiblemente por cambios metabólicos e hipoperfusión asociados a la falla cardiaca; en la edad avanzada se presentan más enfermedades de tipo degenerativo. El desacondicionamiento físico asociado aumenta el imbalance muscular y facilita síndromes por sobreuso como la tendinitis del manguito rotador. La vinculación a una Clínica de Falla Cardiaca con un programa de rehabilitación establecido, permitirá la detección temprana y la intervención adecuada durante el mismo de las patologías musculoesqueléticas asociadas haciendo que disminuya la discapacidad y mejore la calidad de vida de estos pacientes.

\section{Declaración de fuentes de financiación y posibles conflictos de interés}

El presente estudio fue financiado con recursos propios de la Sección de Cardiologia de la Facultad de Medicina de la Universidad de Antioquia.

No hay conflictos de interés.

\section{Referencias}

1. Go AS, Mozaffarian D, Roger VL, Benjamin EJ, Berry JD, Borden WB et al. Heart Disease and Stroke Statistics--2013 Update. Circulation 2013; 127: e6-e245.

2. Van Tol B, Huijsmans RJ, Kroon DW, Schothorst M, Kwakkel G. Effects of exercise training on cardiac performance, exercise capacity and quality of life in patients with heart failure: A meta-analysis. Eur J Heart Fail 2006; 8: 841-850.
3. Braunstein JD, Anderson GF, Gerstenblith G, Weller W, Niefeld M, Herbert R et al. Noncardiac Comorbidity Increases Preventable Hospitalizations and Mortality among Medicare Beneficiaries With Chronic Heart Failure. J Am Col Cardiol 2003, 42 (7): 1226 -33.

4. Hambrecht $\mathbf{R}$, Wolf A, Gielen S, Linke A et al. Effect of exercise on coronary endothelial function in patient with coronary artery disease. $N$ Engl $\mathrm{J}$ Med 2000; 342: 454-60.

5. Hambrecht R, Gielen S, Linke A. et al. Effects of exercise training on left ventricular function and peripheral resistance in patients with chronic heart failure. JAMA 2000; 283: 3095- 3101.

6. Shen J, Perez-Terzic C. Prevalence of Musculoskeletal Disorders in Persons With Diagnosed Cardiovascular Diseases Participating in an Outpatient Cardiac Rehabilitation Program. Arch Phys Med Rehabil 2006; 87: E10.

7. Piña I, Apstein CS, Baldy G, Belardinelli R, Chaitman BR, Duscha BD et al. Exercise and heart failure. A statement from the American Association Committee on exercise, rehabilitation and prevention. Circulation 2003; 107:1210-1225.

8. Jessup M, Brozena S. Heart Failure. N Engl J Med 2003; 348: 2007-18.

9. Felson D. Osteoarthritis of the Knee. N Engl J Med 2006; 354: 841-8.

10. Yamaguchi K, Ditsios K, Middleton WD, Hildebolt CF, Galatz LM, Teefey SA. The demographic and morphological features of rotator cuff disaease. $J$ Bone Surg Am 2006; 88(8): 1699-1704.

11. Wreje U, Brorsson BA. Multicenter randomized controlled trial of injections of sterile water and saline for chronic myofascial pain syndromes. Pain 1995, 61(3): 441-444.

12. Johannes CB, Le TK, Zhou X, Johnston JA, Dworkin RH. The prevalence of chronic pain in United States adults: results of an Internet-based survey. $J$ Pain 2010; 11(11): 1230-1239.

13. Toth M, palmer B, LeWinter $\mathbf{M}$. Effect of heart failure on skeletal muscle myofibrillar protein content, isoform expression and calcium sensitivity. Int $J$ Card 2006, 107: 211-219.

14. Dall'Ago p, et al. Inspiratory muscle training in patients with heart failure and inspiratory muscle weakness. A randomized trial. JACC 2006, 47(4): 757-763.

15. Moser D, et al. Vulnerabilities of patients recovering from an exacerbation of chronic heart failure. Am Heart J 2005, 150 (5): 984.e7-13. 